\title{
26. Theory of conformal mapping of a multiply connected domain, II.
}

\author{
By Masatsugu TsuiI.
}

(Received August 20, 1943.)

Let $F$ be the Riemann surface of an algebraic function of genus $\rho(\geqq 2)$ spread over the $w$-plane and $C_{1}, \ldots, C_{p}$ be $p$ Jordan curves on $F$, which have no common points with each other and do not separat $F$ into two pieces.

We cut $F$ along $C_{1}, \ldots, C_{p}$ and the resulting surface be denoted by $\bar{F}_{0}$. We take infinitely many same samples as $\bar{F}_{0}$ and connect them along the opposite shores of $C_{i}$ in the well known way and obtain a covering surface $F^{(\infty)}$ of $F \cdot F^{(\infty)}$ is of planar character, hence we can map $F^{(\infty)}$ on a schlicht domain $D$ on the $z$-plane by $w=f(z) . \quad D$ is the outside of a certain closed set $E$. In my former paper $\left({ }^{1}\right)$, which we refer as (I) in the following, I have proved:

Theorem IV. E is of capacity zero.

I have found that the proof is incomplete so far as I have used an unproved theorem in the proof $\left({ }^{2}\right)$, so that I will here give another proof of Theorem IV.

I will first prove some lemmas.

\section{Some Lemmas.}

Lemma 1. Let $D$ be a domain in $|\zeta|<1$, such that every point $\zeta$ in $D$ has a distance $\geqq k$ from a point $a$ on $|\zeta|=1$. We map $|\zeta|<1$ on the upper half of tho $n=x+i y$-plane by $\zeta=a \frac{v-i}{v+i}$. Then for any point $\zeta$ in $D$,

$$
\frac{k^{2}}{2} y \leqq 1-|\zeta| \leqq 4 y
$$

Proof. From $\zeta=a \frac{v-i}{v+i}$, we have $1-|\zeta|^{2}=\frac{4 y}{|v+i|^{2}}$. Hence

(1) M. Tsuji : Theory of conformal mapping of a multiply connected domain. This Journ., 18 (1943), 759-775. Mr. K. Arima has given another proof of Theorem IV in Zengoku Sizyô Danwakai, 257 (1943).

(2) I could proved the theorem, which will appear in this Journal, 19 (1944). (Added during the correetion). 


$$
1-|\zeta|=\frac{4 y}{(1+|\zeta|)|v+i|^{2}} \leqq 4 y
$$

and by the hypothesis, $|\zeta-a|=\frac{2}{|v+i|} \geq k$, so that

$$
1-|\zeta|=\frac{4 y}{(1+|\zeta|)|v+i|^{2}} \geq \frac{4 y}{2}\left(\frac{k}{2}\right)^{2}=\frac{k^{2}}{2} y
$$

Lemma 2. Let $G$ be a group of linear transformations, which make the upper half of the $v=x+i y$-plane invariant and $v_{0}$, $v_{0}^{\prime}$ be two points in $\Im v>0$ and $v_{n}, v_{n}^{\prime}$ be the transforms of $v_{0}, v_{0}^{\prime}$ by $G$ respectively. Then $\frac{\widetilde{S} v_{n}^{\prime}}{\mathfrak{S} v_{n}}$ lies between two positive constants independent of the transformations of $G$.

Proof. Let $\frac{|d v|}{y}$ be the hyperbolic line element in $\Im v>0$ and $s_{0}$ be the hyperbolic distance between $v_{0}, v_{0}^{\prime}$, then $s_{0}=\int_{C} \frac{|d v|}{y}$, where $C$ is a part bounded by $v_{0}$ and $v_{0}^{\prime}$ of an orthogonal circle to the real axis through $v_{0}$ and $v_{0}^{\prime}$. Let $\Im v_{n}=y_{n} \leqq \Im v_{n}^{\prime}=y_{n}^{\prime}$ and $C_{n}$ be the transform of $C$ by $G$, then

so that $1 \leqq \frac{y_{n}^{\prime}}{y_{n}} \leqq e^{s_{0}}$.

$$
s_{0}=\int_{C_{n a}} \frac{|d v|}{y} \geqq \int_{y_{n b}}^{y^{\prime}} \frac{d y}{y}=\log \frac{y_{n}^{\prime}}{y_{n}},
$$

Lemma 3. Let $G$ be a group of linear transformations, which make $|z|<1$ invariant, such that its fundamental domain $D_{0}$ with its boundary lies entirely in $|z|<1$. Let $z_{0}$ be a point in $D_{0}$ and $z_{n}$ be its transforms. by $G$. Then $\sum_{n=0}^{\infty}\left(1-\left|z_{n}\right|\right)=\infty$.

Proof. Let $\frac{|d z|}{1-|z|^{2}}$ be the hyperbolic line element in $|z|<1$ and $\sigma_{0},\left|D_{0}\right|$ be the hyperbolic diameter and area of $D_{0}$ respectively. We denote the number of $z_{n}$ in $|z| \leqq r$ by $n(r)$. Let $r<1$ and we determine $r_{0}(<r<1)$ such that

$$
\sigma_{0}=\int_{r_{0}}^{r} \frac{d r}{1-r^{2}}=\frac{1}{2} \operatorname{lcg} \frac{1+r}{1-r} \cdot \frac{1-r_{0}}{1+r_{0}} .
$$

Then, since the transform $D_{n}$ of $D_{0}$ by $G$ has the same hyperbolic diameter $\sigma_{0}$ as $D_{0}$, any $D_{n}$, which msets $|z|=r_{0}$, is contained in $|z| \leqq r$. Hence, since the hyperbolic area $S_{0}$ of a disc: $|z| \leqq r_{0}$ is given by 


$$
S_{0}=\int_{0}^{2 \pi} \int_{0}^{r_{0}} \frac{r d r d \theta}{\left(1-r^{2}\right)^{2}}=\frac{\pi r_{0}^{2}}{1-r_{0}^{2}},
$$

we have

$$
n(r)\left|D_{0}\right| \geqq S_{0}=\frac{\pi r_{0}^{2}}{1-r_{0}^{2}}=\frac{\pi(1+r-\lambda(1-r))^{2}}{4 \lambda(1-r)(1+r)} \geqq \frac{\text { const. }}{1-r},
$$

where $\lambda=e^{2 \sigma_{0}}$.

Hence $N(1)=\int_{\varepsilon}^{1} \frac{n(r)}{r} d r=\infty$, so that $\sum_{n=0}^{\infty}\left(1-\left|z_{n}\right|\right)=\infty, \quad$ q.e.d.

\section{Proof of Theorem IV.}

We assume $p=2$. Let $w=f(z)$ map the outside of $E$ on $F^{(\infty)}$, then $f(z)$ is automorphic with respect to a group $G$ of transformations $U$ : $z^{\prime}=U(z)$, where $z, z^{\prime}$ correspond to the same point on $F$. $U$ transforms the outside of $E$ into itself. Let $\bar{F}_{0}$ be the starting sheet of $F^{(\infty)}$ and let it correspond to $\bar{S}_{\theta}$ on the $z$-plane. $\bar{S}_{0}$ is bounded by four Jordan curves: $\Gamma_{1}, \Gamma_{2}, \Gamma_{3}, I_{4}$, where $\Gamma_{1}, \Gamma_{2} ; \Gamma_{3}, \Gamma_{4}$ correspond to the both shores of $C_{1}$, $C_{2}$ respectively. Let $z_{0}$ be a point in $\bar{S}_{0}$ and we connect $z_{0}$ to a point $z_{i}$ on $I_{i}$ by a Jordan arc $\Lambda_{i}(i=1,2,3,4)$, where $z_{1}, z_{2} ; z_{3}, z_{4}$ correspond to the same point on $C_{1}, C_{2}$ respectively and $A_{i}$ have no common points with each other except $z_{0}$. We cut $\bar{S}_{0}$ along $\Lambda_{1}, \Lambda_{2}, \Lambda_{3}, \Lambda_{4}$ and the resulting domain be denoted by $S_{0}$. If we apply all transformations of $G$ to $S_{0}$ and $\dot{\Gamma}_{i}$, we obtain domains: $S_{0} ; S_{1}, S_{2}, S_{3}, S_{4} ; S_{11}, S_{12}, S_{13} ; S_{21}, S_{22}, S_{23}$; $S_{31}, S_{32}, S_{33} ; S_{41}, S_{42}, S_{43}, \ldots \ldots, S_{i k_{1} \ldots k_{22}}, \ldots$ and curves: $\Gamma_{1}, \Gamma_{2}, \Gamma_{3}, \Gamma_{4} ;$ $\Gamma_{11}, \Gamma_{12}, \Gamma_{13} ; \Gamma_{21}, \Gamma_{22}, \Gamma_{23} ; \Gamma_{31}, \Gamma_{32}, \Gamma_{33} ; \Gamma_{41}, \Gamma_{42}, \Gamma_{43}, \ldots, \Gamma_{i k_{1} \ldots k_{n}}, \ldots$ $\left(i=1,2,3,4 . k_{1}, \ldots, k_{n}=1,2,3\right)$, where $S_{i k_{1} \ldots k_{n}}$ is contained in $S_{i k_{1} \ldots k_{2 n-1}}$ and $\Gamma_{i k_{1} \ldots k_{n}}$ is the outer boundary of $S_{i k_{1} \ldots k_{n}}$ as shown in Fig. 1 in (I).

We put

$$
S=S_{0}+S_{1}+S_{2}+S_{3}+S_{4}+\cdots+S_{i k_{1} \cdots k_{n}}+\cdots
$$

Let $\mathfrak{F}^{(\infty)}$ be the simply connected universal covering Riemann surface of the outside of $E$ and $G^{\prime}$ be the group of transformations, which interchange one sheet of $\mathfrak{F}^{(\infty)}$ into another sheet. Then $S$ is the fundamental domain of $G^{\prime}$ and $S_{0}$ is the fundamental domain of a group $\bar{G}=G+G^{\prime}$.

Next we map $\mathfrak{F}^{(\infty)}$ on $|\zeta|<1$ by $z=\phi(\zeta)$ and let $S, S_{0}, S_{i}, S_{i k_{1} \cdots k_{n}}$ $\left(i=1,2,3,4 . k_{1}, \ldots, k_{n}=1,2,3\right)$ correspond to $D^{(k)}, D_{0}^{(k)}, D_{i}^{(k)}, D_{i k_{1}}^{(k)} \cdots k_{n}$, $(k=0,1,2,3, \ldots)$ in $|\zeta|<1$ respectively, where

$$
\begin{array}{ll}
D^{(0)}=D_{0}^{(0)}+D_{1}^{(0)}+D_{2}^{(0)}+D_{3}^{(0)}+D_{4}^{(0)}+\cdots & +D_{i k_{1} \cdots k_{n 0}}^{(0)}+\cdots \\
D^{(k)}=D_{0}^{(k)}+D_{1}^{(k)}+D_{2}^{(k)}+D_{3}^{(k)}+D_{4}^{(k)}+\cdots & +D_{i k_{1} \cdots k_{n k}}^{(k)}+\cdots
\end{array}
$$


$G, G^{\prime}, \bar{G}$ become groups of transformations in $|\zeta|<1$, which we denote by the same letters. 'Since any transformation of $G, G^{\prime}, \bar{G}$ makes $|\zeta|<1$ invariant, $G, G^{\prime}, \bar{G}$ are groups of linear transformations in $|\zeta|<1$.

$D^{(0)}$ is the fundamental domain of $G^{\prime}$ and $D_{0}^{(0)}$ is the fundamental domain of $\bar{G}$. We take a point $\zeta_{0}$ in $D_{0}^{(0)}$ and let $\zeta_{0}^{(k)}$ be its transform in $D_{0}^{(k)}$ by $G^{\prime}$ and $\zeta_{i}^{(k)}, \zeta_{i k_{1}}^{(k)} \ldots k_{n n}$ be its transform in $D_{i}^{(k)}, D_{i k_{1} \ldots k_{n n}}^{(k)}$ by $G$ respectively, so that

$$
\zeta_{0}^{(k)} \in D_{0}^{(k)}, \quad \zeta_{i}^{(k)} \in D_{i}^{(k)}, \quad \zeta_{i k_{1} \cdots k_{n n}}^{(k)} \in D_{i k_{1} \cdots k_{n a}}^{(k)} .
$$

We will prove that $\sum_{k=0}^{\infty}\left(1-\left|\zeta_{0}^{(k)}\right|\right)=\infty$, which is equivalent to our Theorem.

Now $D_{1}^{(k)}$ is separated from $D_{0}^{(k)}$ by a part of a Jordan arc $L_{1}$, which corresponds to $\Gamma_{1}$ on the $z$ wplane.

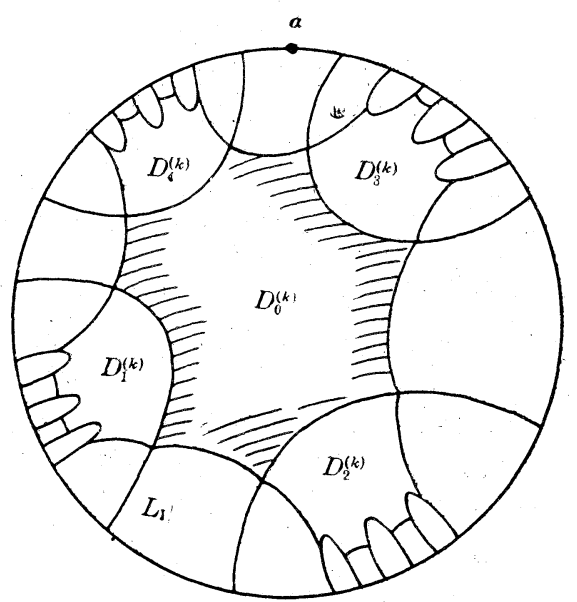

Fig. 1.

$L_{1}$ separates $|\zeta|<1$ into two domains: $\Delta, \Delta^{t}$, where $\Delta$ contains $D_{1}^{(t)}$ in it. Since $\Gamma_{1}$ contains $S_{1 k_{1} \ldots k_{n}}\left(k_{1}, \ldots, k_{n}=1,2,3\right)$ in it, we see that $\Delta$ contains $D_{1 k_{1} \ldots k_{i n}}^{(k)}$.

Since the diameter of $D^{(k)}$ diminishes to zero for $k \rightarrow \infty$, we can find a point $a$ on $|\zeta|=1$, such that for any point $\zeta$ in $\Delta+D_{0}^{(k)},|\zeta-a| \geqq k$, where $k$ is a positive constant independent of the transformations of $G$.

By $\zeta=a \frac{v-i}{v+i}$, we map $|\zeta|<1$ on the upper half of the $v=x+i y$ plane and let $G, G^{\prime}, \bar{G}$ become groups of linear transformations in $\tilde{J} v>0$, which we denote by the same letters. Let

$$
D_{0}^{(k)}, \zeta_{0}^{(k)} ; \quad D_{i}^{(k)}, \zeta_{i}^{(k)} ; \quad D_{i k_{1} \cdots k_{2}}^{(k)}, \quad \zeta_{i k_{1} \cdots k_{n}}^{(k)}
$$


correspond to

$$
\begin{array}{ll}
\sigma_{0}^{(k)}, \quad v_{0}^{(k)}=x_{0}^{(k)}+i y_{0}^{(k)} ; \quad & \sigma_{i}^{(k)}, \quad v_{i}^{(k)}=x_{i}^{(k)}+i y_{i}^{(k)} ; \\
& \sigma_{i k_{1} \cdots k_{r s}, \quad}^{(k)}, v_{i k_{1} \cdots k_{s i}}^{(k)}=x_{i k_{1} \cdots k_{s o}}^{(k)}+i y_{i k_{1} \cdots k_{s h}}^{(k)} .
\end{array}
$$

in $\Im v>0$ respectively, so that

$$
v_{0}^{(k)} \in \sigma_{0}^{(k)}, \quad v_{i}^{(k)} \in \sigma_{i}^{(k)}, \quad v_{i k_{1} \cdots k_{n}}^{(k)} \in \sigma_{i k_{1} \cdots k_{3 i}}^{(k)} .
$$

Since $D_{1 k_{1} \cdots k_{n b}}^{(k)}$ is contained in $\lrcorner$, we have by Lemma 1 ,

$$
\begin{gathered}
\frac{k^{2}}{2} y_{0}^{(k)} \leqq 1-\left|\zeta_{0}^{(k)}\right| \leqq 4 y_{0}^{(k)}, \quad \frac{k^{2}}{2} y_{1}^{(k)} \leqq 1-\left|\zeta_{1}^{(k)}\right| \leqq 4 y_{1}^{(k)}, \\
\frac{k^{2}}{2} y_{1 k_{1} \cdots k_{n_{3}}}^{(k)} \leqq 1-\left|\zeta_{1 k_{1} \cdots k_{n}}^{(k)}\right| \leqq 4 y_{1 k_{1} \cdots k_{n}}^{(k)} .
\end{gathered}
$$

We consider only $\sigma_{1 k_{1} \cdots k_{a}}^{(k)}$ in the following.

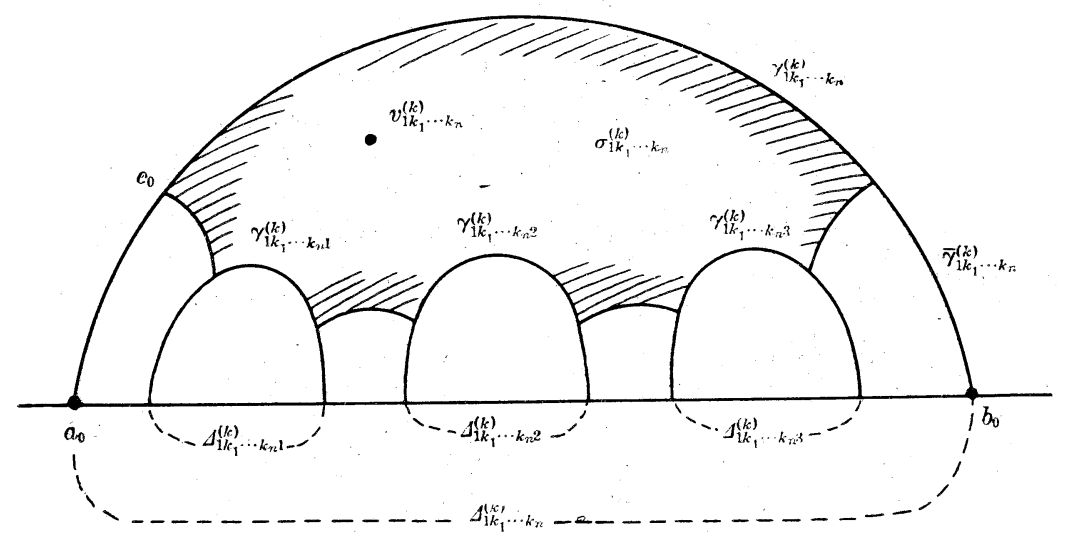

Fig. 2.

Let the boundary of $\sigma_{1 k_{1} \ldots k_{*}}^{(k)}$ contain. Jordan arcs :

$$
\gamma_{1 k_{1} \cdots k_{n}}^{(k)}, \quad \gamma_{1 k_{1} \cdots k_{24} 1}^{(k)}, \quad \gamma_{1 k_{1} \cdots k_{n} 2}^{(k)}, \quad \gamma_{1 k_{1} \cdots k_{n 3} 3}^{(k)},
$$

which are parts of Jordan ares:

$$
\bar{\gamma}_{j k_{1} \cdots k_{n b}}^{(k)}, \quad \bar{\gamma}_{1 k_{1} \cdots k_{n b} 1}^{(k)}, \quad \bar{\gamma}_{1 k_{1} \cdots k_{n} 2}^{(k)}, \quad \bar{\gamma}_{1 k_{1} \cdots k_{n} 3}^{(k)},
$$

which correspond to

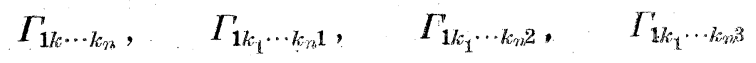

on the $z$-plane. Each curve of (10) ends at two points on the real axis and let the distance between these two points be denoted by 


$$
J_{1 k_{1} \cdots k_{k_{2}}}^{(k)}, \quad J_{1 k_{1} \cdots k_{n_{2} 1} 1}^{(k)}, \quad J_{1 k_{1} \cdots k_{n 2} 2}^{(k)}, \quad J_{1 k_{1} \cdots k_{n 2} 3}^{(k)}
$$

respectively. Then I have proved in (I) :

$$
\sum_{k_{1}}^{1,2,3} J_{k_{n 2}}^{(k)} J_{k_{1} \cdots k_{n 3}} \leqq \frac{1}{(1+\varepsilon)^{n-1}}\left(J_{11}^{(k)}+J_{12}^{(k)}+J_{13}^{(k)}\right)
$$

where $\varepsilon$ is a positive constant independent of the transformations of $\bar{G}$. Hence

$$
\sum_{n=1}^{\infty} \sum_{k_{1} \cdot \cdots, k_{k_{n}}}^{1,2,3} J_{k_{1}}^{(k)} \cdots k_{n_{n}} \leqq \text { const. }\left(J_{11}^{(k)}+J_{12}^{(k)}+J_{13}^{(k)}\right) .
$$

Let $\bar{\gamma}_{1 k_{1} \cdots k_{n b}}^{(k)}$ end at two points $a_{0}, b_{0}$ on the real axis and $c_{0}$ be one end point of $\gamma_{1 k_{1} \cdots k_{2 l}}^{(k)}$. Then since $c_{0}, v_{1 k_{1} \cdots k_{i l}}^{(k)}$ are equivalent by $\bar{G}$ to the corresponding points in $\sigma_{0}^{(0)}$, we have by Lemma 2 ,

$$
\Im v_{1 k_{1} \cdots k_{n b}}^{(k)}=y_{1 k_{1} \cdots k_{n}}^{(k)} \leqq \text { const. } \Im c_{0}
$$

Since $a_{0}, c_{0}, b_{0}$ are equivalent by $\bar{G}$ to the corresponding points similarly obtained from the boundary of $\sigma_{0}^{(0)}$, we see that $a_{0}, c_{0}, b_{0}$ lie on a circular arc, which makes a fixed angle with the real axis, so that

$$
\Im c_{0} \leqq \text { const. }\left(b_{0}-a_{0}\right)=\text { const. } J_{1_{1} k \cdots k_{n}}^{(k)} \text {. }
$$

Hence we have from (14), (15), (16),

$$
\sum_{n=1}^{\infty} \sum_{k_{1}, \cdots, k_{n 2}}^{1.1,3,} y_{1 k_{1} \cdots \cdots k_{n n}}^{(k)} \leqq \text { const. }\left(J_{11}^{(k)}+J_{12}^{(k)}+J_{13}^{(k)}\right)
$$

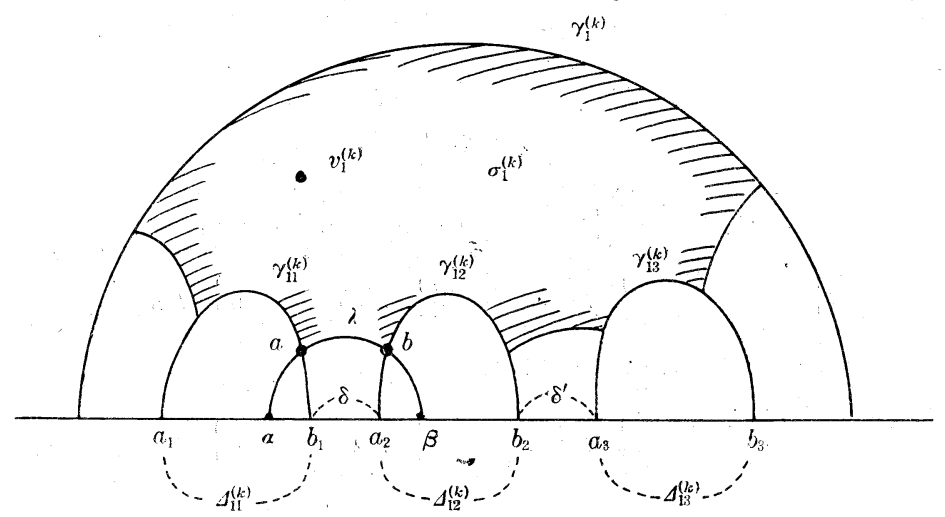

Fig. 3.

Since as $I$ have proved in (I), the ratio of any two numbers of $J_{11}^{(k)}$, $\Delta_{12}^{(k)}, A_{13}^{(k)}, \delta, \delta^{\prime}$ in Fig. 3 lies between two positive constants, we have 


$$
J_{11}^{(k)} \geq \text { const. }\left(J_{11}^{(k)}+J_{12}^{(k)}+J_{13}^{(k)}\right) .
$$

Let $\lambda$ be a boundary $\operatorname{arc}\left({ }^{3}\right)$ of $\sigma_{1}^{(k)}$, such that its one end point $a$ on $\gamma_{11}^{(k)}$ is transformed into another end point $b$ on $\gamma_{12}^{(k)}$ by a transformation $U$ of $\bar{G}$. We apply all transformations $U^{n}(n=0, \pm 1, \pm 2, \ldots)$ to $\lambda$, then we obtain a Jordan are $\bar{\lambda}$ ending at two points $\alpha, \beta$ on the real axis. Since the ratio of any two numbers of $a-a_{1}, b_{1}-\alpha, \beta-b_{1}$ lie between two fixed positive constants and $a_{1}, a, b_{1}$ and $\alpha, a, \beta$ lie on circular ares making fixed angles with the real axis, we have

By Lemma 2,

$$
\left.\Im a \geq \text { const. }\left(b_{1}-a_{1}\right)=\text { const. }\right\lrcorner_{11}^{(k)} \text {. }
$$

$$
\begin{gathered}
\Im a \leqq \text { const. } \Im v_{1}^{(k)}=\text { const. } y_{1}^{(k)}, \\
y_{1}^{(k)} \leqq \text { const. } y_{0}^{(k)} .
\end{gathered}
$$

Hence we have from (17), (18), (19), (20), (21),

Hence by (8)

$$
y_{1}^{(k)}+\sum_{n=1}^{\infty} \sum_{k_{1}, \cdots, k_{n}}^{1,2,3} y_{k_{1}}^{(k)} \cdots k_{n} \leqq \text { const. } y_{0}^{(k)} \text {. }
$$

$$
\left(1-\left|\zeta_{1}^{(k)}\right|\right)+\sum_{n=1}^{\infty} \sum_{k_{1}}^{1, \cdots, k_{k n}}\left(1-\left|\zeta_{1 k_{1} \cdots k_{n a}}^{(k)}\right|\right) \leqq \text { const. }\left(1-\left|\zeta_{0}^{(k)}\right|\right) \text {. }
$$

Similarly we have for $i=1,2,3,4$,

$$
\left(1-\left|\zeta_{i}^{(k)}\right|\right)+\sum_{n=1}^{\infty} \sum_{k_{1}, \ldots, k_{n n}}^{1 \cdot 2,3}\left(1-\left|\zeta_{2 k_{1} \cdots k_{22}}^{(k)}\right|\right) \leqq \text { const. }\left(1-\left|\zeta_{0}^{(k)}\right|\right) \text {. }
$$

Summing up for $i=1,2,3,4$, we have

$$
\left(1-\left|\zeta_{0}^{(k)}\right|\right)+\sum_{i=1}^{4}\left(1-\left|\zeta_{i}^{(k)}\right|\right)+\sum_{i=1}^{4} \sum_{n=1}^{\infty} \sum_{k_{1}, \cdots, k_{n}}^{1,2,3}\left(1-\left|\zeta_{i k_{1} \ldots k_{n}}^{(k)}\right|\right) \leqq \text { const. }\left(1-\left|\zeta_{0}^{(k)}\right|\right) \text {. }
$$

Let $\zeta_{n}$ be the transform of $\zeta_{0}$ by $\bar{G}$, then the left hand side of (22) is nothing but $\sum_{\zeta_{n} \in D^{(k)}}\left(1-\left|\zeta_{n}\right|\right)$, hence we have an important relation :

$$
\sum_{\left.\zeta_{n} \in D^{(l)}\right)}\left(1-\left|\zeta_{n}\right|\right) \leqq \text { const. }\left(1-\left|\zeta_{0}^{(k)}\right|\right)
$$

(3) For example we take $\lambda$ such that it corresponds to the boundary of $S_{0}$ on the $z$-plane, whicn belongs to one of both shores of $\Lambda_{1}$ and $\Lambda_{2}$. 
Hence

$$
\sum_{n=0}^{\infty}\left(1-\left|\zeta_{n}\right|\right)=\sum_{k=0}^{\infty} \sum_{\zeta_{n} \in D^{(k)}}\left(1-\left|\zeta_{n}\right|\right) \leqq \text { const. } \sum_{k=0}^{\infty}\left(1-\left|\zeta_{0}^{(k)}\right|\right)
$$

Since the fundamental domain $D_{0}^{(0)}$ of $\bar{G}$ lies entirely in $|\zeta|<1$, 'we have by Lemma $3, \sum_{n=0}^{\infty}\left(1-\left|\zeta_{n}\right|\right)=\infty$, so that

$$
\sum_{k=0}^{\infty}\left(1-\left|\zeta_{0}^{(k)}\right|\right)=\infty
$$

If $E$ is of capacity positive, then by Frostman's theorem(4) the chracteristic function $T(r)$ of $\phi(\zeta)$ is bounded in $|\zeta|<1$, so that $\sum_{k=0}^{\infty}\left(1-\left|\zeta_{0}^{(k)}\right|\right)<\infty$, which contradicts (24). Hence $E$ is of capacity zero, q. e. d.

Similarly we can prove Theorem VI and VII in my former paper.

$$
\begin{aligned}
& \text { Mathematical Institute, } \\
& \text { Tokyo Imperial University. }
\end{aligned}
$$

(4) Frostman : Potentiel d'equilibre et capacité des ensembles. Lund. (1935). 